Georgian Mathematical Journal

1(1994), No. 3, 315-323

\title{
LIMIT BEHAVIOR OF SOLUTIONS OF ORDINARY LINEAR DIFFERENTIAL EQUATIONS
}

\author{
FRANTIŠEK NEUMAN
}

\begin{abstract}
A classification of classes of equivalent linear differential equations with respect to $\omega$-limit sets of their canonical representatives is introduced. Some consequences of this classification to the oscillatory behavior of solution spaces are presented.
\end{abstract}

\section{INTRODUCTION}

Many authors dealt with the behavior of solutions of differential equations to the (mostly right) end of the interval of definition - the limit behavior (often considered for the independent variable tending to $\infty$ ). Asymptotic, oscillatory and other qualitative properties of solutions of linear differential equations were intensively studied e.g. by N.V.Azbelev and Z.B.Caljuk [1], J.H.Barrett [2], G.D.Birkhoff [3], O.Boruvka [4], W.A.Coppel [5], M.Greguš [6], G.B.Gustafson [7], M.Hanan [8], I.T.Kiguradze and T.A.Chanturia [9], G.Sansone [14], C.A.Swanson [15], and many others.

The aim of this paper is to introduce a certain classification of the limit behavior of solutions of linear differential equations, a classification which is invariant with respect to the most general pointwise transformations of these equations. This classification has natural consequences to the oscillatory and asymptotic behavior of solutions. The main tool is based on the geometric approach introduced in [11] which enables us to convert some "non-compact" problems into "compact" ones. This method was applied for solving some open problems [12], and it has recently been explained systematically in detail together with other methods and results concerning linear differential equations in the monograph [13].

1991 Mathematics Subject Classification. 34A26, 34A30, 34C05, 34C10, 34C11, $34 \mathrm{C} 20$. 


\section{BACKGROUND AND PRELIMINARY RESULTS}

Let $C^{n}(I)$ denote the set of all functions defined on an open interval $I \subseteq \mathbb{R}$ with continuous derivatives up to and including the order $n$. For $n \geq 2$, let $\mathcal{L}_{n}$ stand for all ordinary linear differential equations of the form

$$
P_{n} \equiv y^{(n)}+p_{n-1}(x) y^{(n-1)}+\cdots+p_{0}(x) y=0 \text { on } I,
$$

$I$ being an open interval of the reals, $p_{i}$ are real continuous functions defined on $I$ for $i=0,1, \ldots, n-1$, i.e. $p_{i} \in C^{0}(I), p_{i}: I \rightarrow \mathbb{R}$.

Consider $Q_{n} \in \mathcal{L}_{n}$,

$$
Q_{n} \equiv z^{(n)}+q_{n-1}(t) z^{(n-1)}+\cdots+q_{0}(t) z=0 \quad \text { on } \quad J .
$$

We say that the equation $P_{n}$ is globally equivalent to the equation $Q_{n}$ if there exist two functions,

$$
\begin{gathered}
f \in C^{n}(J), \quad f(t) \neq 0 \text { for each } t \in J, \quad \text { and } \\
h \in C^{n}(J), h^{\prime}(t) \neq 0 \text { for each } t \in J, \quad \text { and } h(J)=I,
\end{gathered}
$$

such that whenever $y: I \rightarrow \mathbb{R}$ is a solution of $P_{n}$ then

$$
z: J \rightarrow \mathbb{R}, \quad z(t):=f(t) \cdot y(h(t)), \quad t \in J,
$$

is a solution of $Q_{n}$.

Let $\mathbf{y}(x)=\left(y_{1}(x), \ldots, y_{n}(x)\right)^{T}$ denote an $n$-tuple of linearly independent solutions of the equation $P_{n}$ considered as a column vector function or as a curve in $n$-dimensional euclidean space $\mathbb{E}_{n}$ with the independent variable $x$ as the parameter and $y_{1}(x), \ldots, y_{n}(x)$ as its coordinate functions; $M^{T}$ denotes the transpose of the matrix $M$.

If $\mathbf{z}(t)=\left(z_{1}(t), \ldots, z_{n}(t)\right)^{T}$ denotes an $n$-tuple of linearly independent solutions of the equation $Q_{n}$, then the global transformation (1) can be equivalently written as

$$
\mathbf{z}(t)=f(t) \cdot \mathbf{y}(h(x))
$$

or, for an arbitrary regular constant $n \times n$ matrix $A$,

$$
\mathbf{z}(t)=A f(t) \cdot \mathbf{y}(h(x))
$$

expressing only that another $n$-tuple of linearly independent solutions of the same equation $Q_{n}$ is taken.

Denote the $n$-tuple $\mathbf{v}=\left(v_{1}, \ldots, v_{n}\right)^{T}$,

$$
\mathbf{v}(x):=\mathbf{y}(x) /\|\mathbf{y}(x)\|,
$$

where $\|\mathbf{y}(x)\|:=\left(y_{1}^{2}(x)+\cdots+y_{n}^{2}(x)\right)^{1 / 2}$ is the euclidean norm of $\mathbf{y}$ in $\mathbb{E}_{n}$. It was shown (see [11] or [13]) that $\mathbf{v} \in C^{n}(I), \mathbf{v}: I \rightarrow \mathbb{E}^{n}$, and the Wronski determinant of $\mathbf{v}$ is different from zero on $I$. Of course, $\|\mathbf{v}(x)\|=1$, i.e. 
$\mathbf{v} \in \mathbb{S}_{n-1}$, where $\mathbb{S}_{n-1}$ is the unit sphere in $\mathbb{E}_{n}$. Denote by $T_{n}$ the differential equation from $\mathcal{L}_{n}$ which has this $\mathbf{v}$ as its $n$-tuple of linearly independent solutions. Evidently $T_{n}$ is globally equivalent to $P_{n}$. Moreover (see again [11] or [13]), if

$$
\mathbf{u}(s):=\mathbf{v}(g(s)),
$$

where the function $g$ satisfies

$$
g(s): J \rightarrow I \subseteq \mathbb{R}, \quad g(J)=I, \quad\left|\left(g^{-1}(x)\right)^{\prime}\right|=\left\|\mathbf{v}^{\prime}(x)\right\|
$$

for the inverse $g^{-1}$ to $g$, and hence $g \in C^{n}(J), g^{\prime}(s) \neq 0$ on $J$, we have $\left\|\mathbf{u}^{\prime}(s)\right\|=1$, i.e. this $\mathbf{u}$ is the length reparametrization of the curve $\mathbf{v}$. Of course, $\|\mathbf{u}(s)\|=\|\mathbf{v}(g(s))\|=1$. If $R_{n}$ denotes the differential equation admitting $\mathbf{u}$ as its $n$-tuple of linearly independent solutions on $J \subseteq \mathbb{R}$, then the above considered equation $P_{n}$ is globally equivalent both to equation $T_{n}$ and to $R_{n}$; equation $R_{n}$ is also called the canonical equation of the whole class of equations from $\mathcal{L}_{n}$ globally equivalent to $P_{n}$. Canonical equations are characterized by admitting $n$-tuples of linearly independent solutions $\mathbf{u}$ satisfying

$$
\|\mathbf{u}(s)\|=1, \quad\left\|\mathbf{u}^{\prime}(s)\right\|=1 ;
$$

for more details see [13].

The following result describes the connection between the behavior of curves $\mathbf{y}, \mathbf{v}$ and $\mathbf{u}$ and the zeros of solutions of the corresponding equations $P_{n}, T_{n}$ and $R_{n}$, see [11] or [13].

Proposition 1. Let $P_{n}, T_{n}$ and $R_{n}$ be equations from $\mathcal{L}_{n}$, and let $\mathbf{y}$, $\mathbf{v}$ and $\mathbf{u}$ denote their $n$-tuples of linearly independent solutions defined as above. For an arbitrary nonzero constant vector $\mathbf{c}=\left(c_{1}, \ldots, c_{n}\right)^{T}$, the solution $\mathbf{c}^{T} \mathbf{y}(x)$ of the equation $P_{n}$ has the zero at $x_{0}$ if and only if the hyperplane

$$
H(\mathbf{c}) \equiv c_{1} \xi_{1}+\cdots+c_{n} \xi_{n}=0 \quad \text { in } \mathbb{E}_{n}
$$

intersects the curve $\mathbf{y}$ at the point of the parameter $x_{0}$.

Moreover, the solution $\mathbf{c}^{T} \mathbf{v}(x)$ of the equation $T_{n}$ has the zero at $x_{0}$ if and only if the great circle $H(\mathbf{c}) \cap \mathbb{S}_{n-1}$ intersects the curve $\mathbf{v}$ at the point of the parameter $x_{0}$. And the solution $\mathbf{c}^{T} \mathbf{u}(s)$ of the equation $R_{n}$ has the zero at $s_{0}=g^{-1}\left(x_{0}\right)$ if and only if the great circle $H(\mathbf{c}) \cap \mathbb{S}_{n-1}$ intersects the curve $\mathbf{u}$ at the point of the parameter $s_{0}$.

In each of the above cases, the order of contact corresponds to the multiplicity of zero. 


\section{Classification of $\omega$-Limit Behavior}

We have seen that a class of globally equivalent equations from $\mathcal{L}_{n}$ is characterized by curve $\mathbf{v} \in \mathbb{S}_{n-1}$, having coordinates in $C^{n}$ with the nonvanishing wronskian. Since the sphere $\mathbb{S}_{n-1}$ is compact, the $\omega$-limit set of $\mathbf{v}$, denoted by $\omega(\mathbf{v})$, is nonempty, closed and connected, see e.g. [10]. Exactly one from the following cases occurs:

$a_{1}: \omega(\mathbf{v})$ is a point $\mathbf{p} \in \mathbb{S}_{n-1}$, i.e. a connected subset of the intersection of a 1-dimensional subspace with $\mathbb{S}_{n-1}$;

$a_{2}: \omega(\mathbf{v}) \subseteq\left(\mathbb{S}_{n-1} \cap \mathbb{E}_{2}\right)$, where $\mathbb{E}_{2}$ is a 2-dimensional subspace of $\mathbb{E}_{n}$, and the case $a_{1}$ is not valid;

$\cdots$

$a_{i}: \omega(\mathbf{v}) \subseteq\left(\mathbb{S}_{n-1} \cap \mathbb{E}_{i}\right)$, where $\mathbb{E}_{i}$ is an $i$-dimensional subspace of $\mathbb{E}_{n}$, and neither from the above cases is valid;

$\ldots$

$a_{n-1}: \omega(\mathbf{v}) \subseteq\left(\mathbb{S}_{n-1} \cap \mathbb{E}_{n-1}\right)$, and neither from the above cases holds;

$a_{n}$ : neither from the above cases is valid.

We will consider also the following subcases of the cases $a_{i}$ for $i=1, \ldots, n$ :

$a_{i}^{0}$ : if the case $a_{i}$ is valid and $\omega(\mathbf{v}) \subseteq \mathbb{S}_{n-1}^{0}$, where $\mathbb{S}_{n-1}^{0}$ is an open hemisphere of $\mathbb{S}_{n-1}$.

Evidently the case $a_{1}$ coincides with $a_{1}^{0}$.

\section{MAIN RESULT}

Theorem. Consider an equation $P_{n}$ from $\mathcal{L}_{n}$; let $T_{n}$ and $R_{n}$ be equations defined as in $\S 2$, and $\mathbf{y}, \mathbf{v}$ and $\mathbf{u}$ denote their $n$-tuples of linearly independent solutions. Let $\omega(\mathbf{v})$ and $\omega(\mathbf{u})$ be the $\omega$-limit sets of $\mathbf{v}$ and $\mathbf{u}$, respectively. If, for some $i$, the case $a_{i}$ is valid for $\mathbf{v}$ (or for $\mathbf{u}$ ), then the same case holds for every equation globally equivalent to $P_{n}$. Moreover, if the subcase $a_{i}^{0}$ is valid for some $i$, then the same subcase is true for every equation globally equivalent to $P_{n}$.

Proof. Suppose first that the case $a_{i}$ is valid for $P_{n} \in \mathcal{L}_{n}$. First it means that $\omega(\mathbf{v}) \subseteq \mathbb{S}_{n-1} \cap \mathbb{E}_{i}$ for $\mathbf{v}:=\mathbf{y} /\|\mathbf{y}\|$. Then for each $\mathbf{z}$,

$$
\mathbf{z}(t):=A f(t) \cdot \mathbf{y}(h(t)),
$$

obtained by a global transformation $\left(1^{\prime \prime}\right)$, we have

$$
\omega(\mathbf{z} /\|\mathbf{z}\|)=\omega(A f \cdot \mathbf{y}(h) /\|A f \cdot \mathbf{y}(h)\|) \subseteq S_{n-1} \cap\left(A \mathbb{E}_{i}\right),
$$

where $A \mathbb{E}_{i}$ is again an $i$-dimensional subspace of $\mathbb{E}_{n}$. Moreover, if $\omega(\mathbf{z} /|\mathbf{z}|) \subseteq$ $S_{n-1} \cap\left(A \mathbb{E}_{j}\right)$ for some $j<i$, we would get the contradiction to our supposition. Hence the case $a_{i}$ is valid for every equation from $\mathcal{L}_{n}$ globally equivalent to $P_{n}$. 
Now suppose that the subcase $a_{i}^{0}$ is valid for $P_{n}$, that means that $\omega(\mathbf{v}) \subseteq$ $\mathbb{S}_{n-1}^{0} \cap \mathbb{E}_{i}$ for $\mathbf{v}:=\mathbf{y} /\|\mathbf{y}\|$. Then for each $\mathbf{z}, \mathbf{z}(t):=A f(t) \cdot \mathbf{y}(h(t))$, we have $\omega(\mathbf{z} /\|\mathbf{z}\|) \subseteq \hat{S}_{n-1}^{0} \cap\left(A \mathbb{E}_{i}\right)$, where $\hat{S}_{n-1}^{0}=\left\{\mathbf{s} ; \mathbf{s}=A \mathbf{r} /\|A \mathbf{r}\|, \mathbf{r} \in S_{n-1}^{0}\right\}$ is again an open hemisphere in $\mathbb{E}_{n}$ and $A \mathbb{E}_{i}$ is an $i$-dimensional subspace of $\mathbb{E}_{n}$. Hence the case $a_{1}^{0}$ is valid for every equation from $\mathcal{L}_{n}$ globally equivalent to $P_{n}$.

Remark 1. This theorem also shows that we may speak about the above cases and subcases with respect to a given equation and not only with respect to a particular $n$-tuple of its solutions, because, due to an arbitrary matrix $A$ in $\left(1^{\prime \prime}\right)$, these cases and subcases are characterized by the properties which are invariant with respect to a choice of an $n$-tuple of linearly independent solutions of the considered equation.

\section{Consequences}

Oscillation or nonoscillation will be always considered with respect to the right end of the definition interval of a considered equation.

Corollary 1. (Oscillatory behavior of solutions). If the case $a_{1}$ is valid for $P_{n} \in \mathcal{L}_{n}$, then there do not exist $n$ linearly independent oscillatory solutions (for $t \rightarrow b_{-}$) of $P_{n}$. Moreover, there exist $n$ linearly independent nonoscillatory solutions of $P_{n}$ as $\left(t \rightarrow b_{-}\right)$.

Proof. Let $P_{n}$ be a given equation, and $\mathbf{y}$ denote an $n$-tuple of its linearly independent solutions. Suppose that there exist $n$ linearly independent oscillatory solutions of $P_{n}$. Then, due to Proposition 1, there are $n$ great circles on $\mathbb{S}_{n-1}$, not containing a common point, each of them being intersected by $\mathbf{v}=\mathbf{y} /\|\mathbf{y}\|$, or equivalently, by $\mathbf{u}$ (see notation in $\S 2$ ) at points with infinitely many parameters to the right end of the interval of definition. Hence on each of these great circles there is at least one point belonging to $\omega(\mathbf{v})(\omega(\mathbf{u}))$. Under our assumption, the case $a_{1}$ is valid for $P_{n}$, i.e. $\omega(\mathbf{v})$ is a single point, say $\mathbf{p}$ on $\mathbb{S}_{n-1}$. Thus this point must be common to $n$ considered circles, which is a contradiction to the linear independence of the solutions. Hence there do not exist $n$ linearly independent oscillatory solutions of $P_{n}$.

Now choose $n$ independent vectors $\mathbf{c}_{1}, \ldots, \mathbf{c}_{n}$ in $\mathbb{E}_{n}$ such that the hyperplanes $H\left(\mathbf{c}_{i}\right), i=1, \ldots, n$ do not go through the point $\mathbf{p}$. Then each solution $\mathbf{c}_{i}^{T} \cdot \mathbf{y}(x)$ is nonoscillatory. In fact, if $\mathbf{c}_{i}^{T} \cdot \mathbf{y}(x)$ were oscillatory, then $\mathbf{y} /\|\mathbf{y}\| \cap H\left(\mathbf{c}_{i}\right)$ would be an infinite sequence on the great circle $\mathbb{S}_{n-1} \cap H\left(\mathbf{c}_{i}\right)$ that should have an accumulation point in $\omega(\mathbf{y} /\|\mathbf{y}\|)=\mathbf{p}$, contrary to our choice of the hyperplanes. 
Corollary 2. (Asymptotic behavior of solutions). If the case $a_{1}$ is valid for equation $P_{n}$ from $\mathcal{L}_{n}$, then $P_{n}$ admits an $n$-tuple $\mathbf{y}^{*}=\left(y_{1}^{*}, \ldots, y_{n}^{*}\right)^{T}$ of linearly independent solutions such that

$$
\lim _{x \rightarrow b_{-}} \frac{y_{1}^{*}}{\sqrt{\left(y_{1}^{*}\right)^{2}+\cdots+\left(y_{n}^{*}\right)^{2}}}=1
$$

and

$$
\lim _{x \rightarrow b_{-}} \frac{y_{i}^{*}}{\sqrt{\left(y_{1}^{*}\right)^{2}+\cdots+\left(y_{n}^{*}\right)^{2}}}=0 \text { for } i=2, \ldots, n .
$$

Proof. In the case $a_{1}$ we have $\lim _{x \rightarrow b_{-}} \mathbf{y}(x) /\|\mathbf{y}(x)\|=\mathbf{p}, \mathbf{p}$ being a point on $\mathbb{S}_{n-1}$. Choose an $n$-tuple of orthonormal vectors $\mathbf{c}_{1}, \ldots, \mathbf{c}_{n}$, where $\mathbf{c}_{1}:=$ $\mathbf{p}$, otherwise arbitrary. Denote by $C$ the orthogonal matrix $\left(\mathbf{c}_{1}, \ldots, \mathbf{c}_{n}\right)$. Define $y_{i}^{*}:=\mathbf{c}_{i}^{T} \cdot \mathbf{y}$, i.e. $\mathbf{y}^{*}=C^{T} \cdot \mathbf{y}$. Then

$$
\begin{gathered}
\lim _{x \rightarrow b_{-}} y_{1}^{*} /\left\|\mathbf{y}^{*}\right\|=\lim _{x \rightarrow b_{-}} \frac{\mathbf{c}_{1}^{T} \mathbf{y}}{\left\|\mathbf{y}^{T} C C^{T} \mathbf{y}\right\|}=\mathbf{c}_{1}^{T} \cdot \lim _{x \rightarrow b_{-}} \mathbf{y} /\|\mathbf{y}\|= \\
\mathbf{c}_{1}^{T} \cdot \mathbf{p}=\mathbf{c}_{1}^{T} \cdot \mathbf{c}_{1}=1
\end{gathered}
$$

and for $i=2, \ldots, n$,

$$
\begin{gathered}
\lim _{x \rightarrow b_{-}} y_{i}^{*} /\left\|\mathbf{y}^{*}\right\|= \\
\lim _{x \rightarrow b_{-}} \frac{\mathbf{c}_{i}^{T} \mathbf{y}}{\left|\mathbf{y}^{T} C C^{T} \mathbf{y}\right|}=\mathbf{c}_{i}^{T} \cdot \lim _{x \rightarrow b_{-}} \mathbf{y} /\|\mathbf{y}\|= \\
\mathbf{c}_{i}^{T} \cdot \mathbf{p}=\mathbf{c}_{i}^{T} \cdot \mathbf{c}_{1}=0 .
\end{gathered}
$$

Corollary 3. If the second order equation

$$
y^{\prime \prime}+p_{1}(x) y^{\prime}+p_{0}(x) y=0 \quad \text { on } \quad I=(a, b), \quad-\infty \leq a<b \leq \infty
$$

is nonoscillatory (for $x \rightarrow b_{-}$), then the case $a_{1}$ is valid for (2). If the equation (2) is oscillatory (for $x \rightarrow b_{-}$), then the case $a_{2}$ holds for (2). The subcase $a_{2}^{0}$ cannot occur.

Proof. For two linearly independent solutions $y_{1}, y_{2}$ of equation (2), $\mathbf{y}=$ $\left(y_{1}, y_{2}\right)^{T}$, the curve $\mathbf{v}=\mathbf{y} /\|\mathbf{y}\|$ is an arc on the unit circle $\mathbb{S}_{1}$ in the plane $\mathbb{E}_{2}$. Due to Proposition 1, if equation (2) is oscillatory for $x \rightarrow b_{-}$, then this arc $\mathbf{v}$ infinitely many times encircles the origin (without turning points, see [13]), and hence $\omega(\mathbf{v})$ is exactly $\mathbb{S}_{1}$. If equation (2) is nonoscillatory for $x \rightarrow b_{-}$, then the arc $\mathbf{v}$ ends by approaching a point on $\mathbb{S}_{1}$, exactly its $\omega$-limit set, and the case $a_{1}$ holds for (2).

Corollary 4. If the case $a_{j}$ is valid for an equation $P_{n}$ for some $j>1$, then there exist $n$ linearly independent oscillatory solutions of $P_{n}$. 
Proof. In the case $a_{j}$ for some $j>1$, the set $\omega(\mathbf{v})$ contains two different points on $\mathbb{S}_{n-1}$, say $\mathbf{p}_{1}$ and $\mathbf{p}_{2}$. Evidently, there exist $n$ hyperplanes $H\left(\mathbf{c}_{i}\right)$, $i=1, \ldots, n$, in $\mathbb{E}_{n}$ with linearly independent vectors $\mathbf{c}_{1}, \ldots, \mathbf{c}_{n}$, each of them separating points $\mathbf{p}_{1}$ and $\mathbf{p}_{2}$ into opposite open halfspaces of $\mathbb{E}_{n}$, i.e. $\mathbf{c}_{i}^{T} \mathbf{p}_{1}>0$ and $\mathbf{c}_{i}^{T} \mathbf{p}_{2}<0$ for each $i=1, \ldots, n$. Hence, due to Proposition 1 , each solution $\mathbf{c}_{i}^{T} \mathbf{y}(x)$ oscillates for $x \rightarrow b_{-}$, because the curve $\mathbf{v}$ intersects infinitely many times the hyperplane $H\left(\mathbf{c}_{i}\right)$ as $x \rightarrow b_{-}$.

Remark 1. As an immediate consequence of this corollary we may state:

If equation $L_{n}$ does not admit $n$ linearly independent oscillatory solutions, then the case $a_{1}$ is valid for it. In particular, if each solution of equation $P_{n}$ is nonoscillatory, then the case $a_{1}$ takes place for $L_{n}$.

Corollary 5. If, for some $i=1, \ldots, n$, the case $a_{i}^{0}$ is valid for equation $P_{n}$, then there exist $n$ linearly independent nonoscillatory solutions of $P_{n}$.

Proof. Let $\mathbf{y}$ denote an $n$-tuple of linearly independent solutions of $P_{n}$. Under our assumption, $\omega(\mathbf{y} /\|\mathbf{y}\|)$ lies inside an open hemisphere of $\mathbb{S}_{n-1}$ determined by a hyperplane $H(\mathbf{p})$. Evidently $\mathbf{p}^{T} \mathbf{y}(x)$ is a nonoscillatory solution. Moreover, $\omega(\mathbf{y} /\|\mathbf{y}\|)$ is closed, and hence there exists an neighbourhood $N$ of the point $\mathbf{p} \in \mathbb{S}_{n-1}$ such that $H(\mathbf{q}) \cap \omega(\mathbf{y} /\|\mathbf{y}\|)=\varnothing$ for each $\mathbf{q} \in N$. If we take $n$ linearly independent vectors (points) $\mathbf{q}_{1}, \ldots, \mathbf{q}_{n}$ from $N$, then

$$
y_{i}:=\mathbf{q}_{i}^{T} \mathbf{y}, \quad i=1, \ldots, n,
$$

are required nonoscillatory solutions. In fact, if one of these solutions were oscillatory, then, again due to Proposition 1, the corresponding hyperplane would intersect the curve $\mathbf{y}$ (or equivalently $\mathbf{y} /\|\mathbf{y}\|$ ) infinitely many times. Hence this hyperplane would contain at least one point in $\omega(\mathbf{y} /\|\mathbf{y}\|)$, contrary to our choice of the above hyperplanes.

Remark 2. Comparing Corollaries 4 and 5 we see that in the case $a_{i}^{0}$ with $i>1$ for $L_{n}$, this equation admits both an $n$-tuple of oscillatory solutions and, at the same time, another $n$-tuple of nonoscillatory solutions.

Remark 3. Also other (e.g. topological) properties of $\omega(\mathbf{v})$ that are invariant with respect to the centroaffine transformations can be considered for introducing other, more detailed classifications of the classes of equivalent linear differential equations from $\mathcal{L}_{n}$. 


\section{ExAmples}

1. The differential equation

$$
y^{(n)}=0 \quad \text { on }(0, \infty)
$$

has $n$ linearly independent solutions: $x^{n-1}, x^{n-2}, \ldots, 1$. For this equation the case $a_{1}$ holds, no solution is oscillatory and

$$
\begin{gathered}
\lim _{x \rightarrow \infty} \frac{x^{n-1}}{\sqrt{\sum_{j=0}^{n-1} x^{2 j}}}=1, \\
\lim _{x \rightarrow \infty} \frac{x^{n-2}}{\sqrt{\sum_{j=0}^{n-1} x^{2 j}}}=0, \ldots, \lim _{x \rightarrow \infty} \frac{1}{\sqrt{\sum_{j=0}^{n-1} x^{2 j}}}=0,
\end{gathered}
$$

in accordance with Corollary 2 and Remark 1.

2. The equation

$$
y^{\prime \prime \prime}+2 y^{\prime \prime}+2 y^{\prime}=0 \quad \text { on }(0, \infty)
$$

admits the solutions: $1, e^{-x} \sin x, e^{-x} \cos x$. For this equation the case $a_{1}$ is valid. There are two linearly independent oscillatory solutions as $x \rightarrow \infty$, there are no three linearly independent oscillatory solutions. This equation admits three linearly independent nonoscillatory solutions, and

$$
\lim _{x \rightarrow \infty} \frac{1}{\sqrt{1+e^{-2 x}}}=1, \quad \lim _{x \rightarrow \infty} \frac{e^{-x} \sin x}{\sqrt{1+e^{-2 x}}}=0, \quad \lim _{x \rightarrow \infty} \frac{e^{-x} \cos x}{\sqrt{1+e^{-2 x}}}=0,
$$

as Corollaries 1 and 2 state.

3 . However, the equation

$$
y^{\prime \prime \prime}-2 y^{\prime \prime}+2 y^{\prime}=0 \quad \text { on } \quad(0, \infty)
$$

admits the solutions: $1, e^{x} \sin x, e^{x} \cos x$; the corresponding $\omega$-limit set is a great circle on the sphere $\mathbb{S}_{2}$ in $\mathbb{E}_{3}$ and hence the case $a_{2}$ is valid for it. However, the subcase $a_{2}^{0}$ does not take place. Except of the constant solutions, each other solution is oscillatory (as $x \rightarrow \infty$ ), see Corollaries 4,5 and Remark 2.

\section{REFERENCES}

1. N.V.Azbelev and Z.B.Caljuk, To the question of distribution of zeros solutions of third order linear differential equation. (Russian) Mat. Sb. (N.S.) $\mathbf{5 1}(1960), 475-486$.

2. J.H.Barrett, Oscillation theory of ordinary differential equations. Adv. in Math. 3(1969), 451-509. 
3. G.D.Birkhoff, On the solutions of ordinary linear homogeneous differential equations of the third order. Ann. of Math. 12(1910/11), 103-127.

4. O.Boruvka, Linear differential transformations of the second order. The English Univ. Press, London, 1971.

5. W.A.Coppel, Disconjugacy. Lecture Notes in Math. 220, Springer, Berlin, 1971.

6. M.Greguš, Linear differential equations of the third order. North Holland, Reidel Co,. Dordrecht-Boston-Lancaster, 1986.

7. G.B.Gustafson, Higher order separation and comparison theorems, with applications to solution space problems. Ann. Mat. Pura Appl. (4) 95(1973), 245-254.

8. M.Hanan, Oscillation criteria for third order linear differential equations. Pacific J. Math. 11(1961), 919-944.

9. I.T.Kiguradze and T.A.Chanturija, Asymptotic properties of solutions of nonautonomous ordinary differential equations. (Russian) "Nauka", Moscow, 1990.

10. V.V.Nemytskii and V.V.Stepanov, Qualitative theory of differential equations. (Russian) "Gostekhizdat", Moscow-Leningrad, 1949.

11. F.Neuman, Geometrical approach to linear differential equations of the $n$-th order. Rend. Mat. 5(1972), 579-602.

12. F.Neuman, On two problems about oscillation of linear differential equations of the third order. J. Diff. Equations 15(1974), 589-596.

13. F.Neuman, Global properties of linear differential equations. Kluwer Acad. Publ. (Mathematics and Its Applications, East European Series 52) \& Academia, Dordrecht-Boston-London \& Praha, 1991.

14. G.Sansone, Equazioni differenziali nel campo reale. Zanichelli, Bologna, 1948.

15. C.A.Swanson, Comparison and oscillation theory of linear differential equations. Academic Press, New York-London, 1968.

(Received 06.04.1993)

Author's address:

Mathematical Institute,

Academy of Sciences of the Czech Republik,

Mendelovo nám. 1, CR-66 282 Brno,

Czech Republik 